\title{
Clinical spectrum of primary adrenal lymphoma: results of a multicenter cohort study
}

\author{
Fatemeh Majidi', Samuela Martino ${ }^{1}$, Mustafa Kondakci' ${ }^{1}$ Christina Antke ${ }^{19}$, Matthias Haase ${ }^{2}$, \\ Vasileios Chortis ${ }^{3,4}$, Wiebke Arlt ${ }^{3,4}$, Cristina L Ronchi ${ }^{4,5}$, Martin Fassnacht $\mathbb{D}^{5,6}$, Claire Laurent ${ }^{7}$,

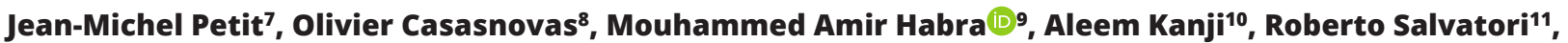 \\ An Thi Nhat Ho ${ }^{20}$, Ariadni Spyroglou ${ }^{12}$, Felix Beuschlein ${ }^{12,13}$, Diego Villa ${ }^{14}$, Wasithep Limvorapitak 14,15 , \\ Björn Engelbrekt Wahlin ${ }^{16}$, Oliver Gimm ${ }^{17}$ Martina Rudelius ${ }^{18}$, Matthias Schott ${ }^{2}$, Ulrich Germing ${ }^{1}$, Rainer Haas ${ }^{1}$ \\ and Norbert Gattermann' ${ }^{1}$
}

\begin{abstract}
'Department of Hematology, Oncology and Clinical Immunology, Heinrich Heine University Düsseldorf, Düsseldorf, Germany, ${ }^{2}$ Department of Endocrinology, Heinrich Heine University Düsseldorf, Düsseldorf, Germany, ${ }^{3}$ Center for Endocrinology, Diabetes and Metabolism, Birmingham Health Partners, Birmingham, UK, ${ }^{4}$ Institute of Metabolism and Systems Research, University of Birmingham, Birmingham, UK, ${ }^{5}$ Divison of Endocrinology and Diabetes, Department of Internal Medicine, University Hospital, University of Würzburg, Würzburg, Germany, ${ }^{6}$ Comprehensive Cancer Center Mainfranken, University of Würzburg, Würzburg, Germany, ${ }^{7}$ University Hospital of Dijon, Dijon, France, ${ }^{8}$ Department of Hematology, University Hospital of Djion, Dijon, France, ${ }^{9}$ Department of Endocrine Neoplasia and Hormonal Disorders, MD Anderson Cancer Center, Houston, Texas, USA, ${ }^{10}$ Baylor College of Medicine, Houston, Texas, USA, ${ }^{11}$ Division of Endocrinology, Diabetes and Metabolism, Johns Hopkins University, Baltimore, Maryland, USA, ${ }^{12}$ Klinik für Endokrinologie und Klinische Ernährung, Universitätsspital Zürich, Zürich, Switzerland, ${ }^{13}$ Klinik und Poliklinik IV, Klinikum der Universität München, Ludweig-Maximilians-Universität München, Munich, Germany, ${ }^{14} \mathrm{BC}$ Cancer Centre for Lymphoid Cancer and University of British Columbia, Vancouver, Canada, ${ }^{15}$ Division of Hematology, Department of Internal Medicine, Thammasat University, Pathumthani, Thailand, ${ }^{16}$ Department of Medicine, Unit of Hematology, Karolinska Institute, Stockholm, Sweden, ${ }^{17}$ Departments of Surgery, and Department of Biomedical and Clinical Sciences, Linköping University, Linköping, Sweden, ${ }^{18}$ Institute of Pathology, Ludweig-Maximilians-Universität München, München, Germany, ${ }^{19}$ Klinik für Nuklearmedizin, Heinrich Heine Universität Düsseldorf, Düsseldorf, Germany, and ${ }^{20}$ Department of Medicine, Medstar Harbor Hospital, Baltimore, Maryland, USA
\end{abstract}

Correspondence should be addressed to F Majidi Email fatemeh.majidi@med. uni-duesseldorf.de

\begin{abstract}
Purpose: We sought to refine the clinical picture of primary adrenal lymphoma (PAL), a rare lymphoid malignancy with predominant adrenal manifestation and risk of adrenal insufficiency.

Methods: Ninety-seven patients from 14 centers in Europe, Canada and the United States were included in this retrospective analysis between 1994 and 2017.

Results: Of the 81 patients with imaging data, 19 (23\%) had isolated adrenal involvement (iPAL), while 62 (77\%) had additional extra-adrenal involvement (PAL+). Among patients who had both CT and PET scans, 18FDG-PET revealed extra-adrenal involvement not detected by CT scan in $9 / 18$ cases (50\%). The most common clinical manifestations were B symptoms (55\%), fatigue (45\%), and abdominal pain (35\%). Endocrinological assessment was often inadequate. With a median follow-up of 41.6 months, 3-year progression-free (PFS) and overall (OS) survival rates in the entire cohort were $35.5 \%$ and $39.4 \%$, respectively. The hazard ratios of IPAL for PFS and OS were 40.1 (95\% Cl: 2.63-613.7, $P=0.008$ ) and 2.69 (95\% Cl: $0.61-11.89, P=0.191$ ), respectively. PFS was much shorter in IPAL vs PAL+ (median 4 months vs not reached, $P=0.006$ ), and OS also appeared to be shorter (median 16 months vs not reached), but the difference did not reach statistical significance $(P=0.16)$. Isolated PAL was more frequent in females $(O R=3.81 ; P=0.01)$ and less frequently associated with $B$ symptoms $(O R=0.159 ; P=0.004)$. Conclusion: We found unexpected heterogeneity in the clinical spectrum of PAL. Further studies are needed to clarify whether clinical distinction between PAL and PAL+ is corroborated by differences in molecular biology.
\end{abstract}




\section{Introduction}

Primary adrenal lymphoma (PAL) is a rare entity that seems to have a poor prognosis. Patients may not only suffer from lymphoma-related symptoms such as fever, night sweats and weight loss (B symptoms), and abdominal or lumbar pain, but have also been reported to develop life-threatening adrenal insufficiency in case of bilateral involvement $(1,2)$. Therefore, a multidisciplinary approach is needed, involving oncologists and endocrinologists. However, due to the rarity of PAL, most oncologists and endocrinologists do not have much experience in treating this disease. Knowledge of PAL is based on less than 250 cases reported in the retrievable scientific literature; the largest series includes 31 patients $(2,3)$.

The diagnostic work-up for PAL includes imaging studies like ultrasonography, CT, MRI and PET, but histopathology is required to confirm the diagnosis (2, 4). This does not conflict with the recommendation that adrenal biopsy should only be performed if the expected findings are likely to alter the management of the individual patient and after biochemical exclusion of catecholamine-producing tumors to prevent potentially life-threatening complications $(5,6)$. A comprehensive endocrine work-up is also strongly recommended according to recent guidelines (7). As the exact cause of an adrenal mass is difficult to ascertain on the basis of imaging characteristics, some PALs are diagnosed only after surgical removal. This should be avoided, because surgery exposes the patient to additional risks, including a considerable delay in starting chemotherapy.

In general, treatment of PAL follows the principles of therapy for B cell non-Hodgkin lymphomas. Reliable prognostic factors have not been identified. Controversial results have been reported as to the prognostic impact of tumor stage, bilateral vs unilateral involvement, involvement of other organs, presence of adrenal insufficiency, serum lactate dehydrogenase (LDH) level, and achieving complete remission after immunochemotherapy $(1,3)$. We present a multicenter case series of 97 patients with PAL, focusing on clinical presentation, radiographic features, immunohistochemistry, treatment results, and prognosis.

\section{Patients and methods}

\section{Data collection}

Fourteen academic medical centers in Europe, Canada and the USA contributed to this study. Ninety-seven patients with PAL were identified between 1994 and 2017. As explained by Rashidi and Fisher (2) in their systematic review of PAL, 'primary adrenal lymphoma is histologically proven lymphoma of one or both adrenal glands in patients with no prior history of lymphoma. If other organs or lymph nodes besides the adrenal glands are involved, the adrenal lesion must be unequivocally dominant'. This definition of PAL is generally used and was therefore adopted in our retrospective multicenter study.

With approval from each participating centers' institutional review boards, retrospective clinical data, laboratory results, imaging and histopathology reports were extracted from patients' medical records and pathology databases. Most of the participating centers are part of the ENS@T registry (European Network for the Study of Adrenal Tumors), which has been approved by the local ethics committees. The study was approved by the following ethics committees: Heinrich Heine Universität Düsseldorf (Ethikkommission der Medizinischen Fakultät); University of Birmingham, Universität Würzburg (Ethik-Kommission bei der Medizinischen Fakultät); Comite de protection des personnes Est1 for university hospitals Dijon, Besancon, Lyon, Strasbourg and Nancy in France; Institutional Review Board at MD Anderson Cancer Center; Johns Hopkins University (Office of Human Subjects Research, Institutional Review Board); Ethikkommission bei der Medizinischen Fakultät der Ludwig-Maximilians-Universität München; British Columbia Cancer Agency Research Ethics Board; Karolinska Institute Ethics Committee, Stockholm; and Universitetssjukhuset Linköping (Regionala ethicprövningsnämnden BESLUT I Linköping).

Due to the retrospective design of the study, informed consent could not be obtained from most patients. Regarding imaging studies, data collection was restricted to the retrieval of radiology reports. Images for repeat measurements were rarely available. Data were entered into a common study-specific data capture sheet by local investigators. The database included patient demographics, clinical presentation, laboratory findings, for example, serum adrenocorticotrophin (ACTH) level, baseline cortisol level, ACTH stimulating test, lactate dehydrogenase (LDH) and Epstein-Barr virus DNA, imaging results (CT and PET scans), histopathological analysis including immunohistochemistry (proliferation marker Ki-67 and lymphoma markers including CD3, CD5, CD10, CD20, CD79a, BCL2, BCL6 and MUM1), treatment data, and clinical follow-up. PAL was defined as histologically proven extra-nodallymphoma that primarily affects the adrenal gland(s) (3). If there was involvement 
of other organs and/or lymph nodes, the diagnosis of PAL was accepted only in the case of unequivocal dominant involvement of the adrenal gland(s) (3). We divided the disease into two subtypes: cases with synchronous extraadrenal involvement at diagnosis (PAL+) and cases with isolated involvement of the adrenal(s) (iPAL). While there is no unmistakeable evidence of adrenal origin in cases with additional extra-adrenal involvement (PAL+), these cases were deemed primary adrenal by treating physicians in the centers, based on clinical and radiological findings.

In the entire study cohort of 97 patients with histopathologically proven PAL, imaging data was available for 81 , detailed patient history for 71 , information about treatment modality for 69 , data on initial treatment response for 62 , and follow-up information for 59 patients.

\section{Statistical analysis}

Survival data were collected until 31 April 2018. Patient characterstics were reported with median and range for continuous variables and as frequencies (\%) for categorical variables. The univariable association of categorical variables was evaluated using Fisher's exact test. Overall survival (OS) was calculated with the nonparametric method of Kaplan-Meier, considering the date of first diagnosis and either the time of death (complete data), irrespective of cause, or the time of last follow-up (censored data). Progression-free survival (PFS) was defined as the time from the date of diagnosis until the date of progression or death from any cause and calculated in the same manner as OS. Maximum observation time was 10 years. In the Kaplan-Meier analysis, comparison between survival curves was made according to the log-rank test. Univariable analysis using Cox proportional model was carried out to identify factors associated with OS and PFS. Because the number of complete datasets appeared too small, we refrained from multivariable analysis. All tests were two sided and $P$-values less than 0.05 were considered statistically significant. All data were analyzed with IBM SPSS statistics software version 24 .

\section{Results}

\section{Clinical and laboratory findings}

The study cohort included 68 males (70\%) and 29 females (30\%). Bilateral adrenal involvement was reported in $49 \%$ of the patients (44/90, missing data in 7 ). As the clinical information was captured mostly from tertiary referral centers, the exact date of first diagnosis and the duration of survival were not available for all patients. Detailed records of clinical manifestations were available in 71 patients. B symptoms, fatigue, abdominal or back pain, and anorexia were the most common symptoms. Shortness of breath/exertional dyspnea, hypotension, and pruritus were less common (Fig. 1). Adrenal insufficiency may have contributed to the patients' symptoms, particularly fatigue and hypotension. It is difficult for the clinician, though, to attribute these symptoms to adrenal insufficiency, as fatigue is commonly found in patients with malignant lymphoma and hypotension may be part of an infectious complication.

PAL was an incidental finding on CT scan in 10 of 71 cases $(14 \%)$ detected during clinical workup for other disorders. Five patients had a history of immune dysfunction, related to human immunodeficiency virus (HIV) infection (2/71) or autoimmune disease (rheumatoid arthritis (2/71), Evans syndrome (1/71)). A concurrent or past diagnosis of cancer was noted in 13 of 71 cases (18\%), in particular prostate cancer (7/51 male patients), breast cancer (1/24 female patients), non-melanoma skin cancer (3/71), and chronic lymphocytic leukemia (2/71). Serum LDH was increased in 53 of $65(82 \%)$ patients tested. A test for Epstein-Barr virus DNA in the serum was done in 30 patients and was positive in 22 cases (73\%). Thirtyseven patients underwent measurement of baseline cortisol level and/or an ACTH stimulation test $(n=13)$. In 20 of these patients, bilateral adrenal involvement was present. Adrenal insufficiency was detected in 14 (70\%) of 20 patients with a bilateral mass, but none of 17 patients with unilateral PAL.

Of the 81 patients with available imaging data, 19 (23\%) had iPAL, while 62 (77\%) had PAL+. The sex distribution (f:m) was 53\%:47\% for iPAL and 22\%:78\% for PAL+. Using the Fisher's exact test, we found a significant association of female sex with the iPAL phenotype (odds ratio: $3.81,95 \%$ CI: $1.294-11.213, P=0.01)$. Median age at diagnosis was 66 years (range: $25-89$ ), with no significant difference between PAL+ and iPAL. Interestingly, isolated PAL was significantly less frequently associated with $B$ symptoms at diagnosis $(\mathrm{OR}=0.159 ; P=0.004)$.

\section{Histopathological diagnosis}

B cell non-Hodgkin lymphoma was the most common histopathological finding, reported in 91\% (88/97) of patients. The most frequent subtype was diffuse large B cell lymphoma (DLBCL) (74/97) including one $T$ cell/histiocyte-rich B cell lymphoma, an uncommon morphological variant of DLBCL. As shown in Table 1, 


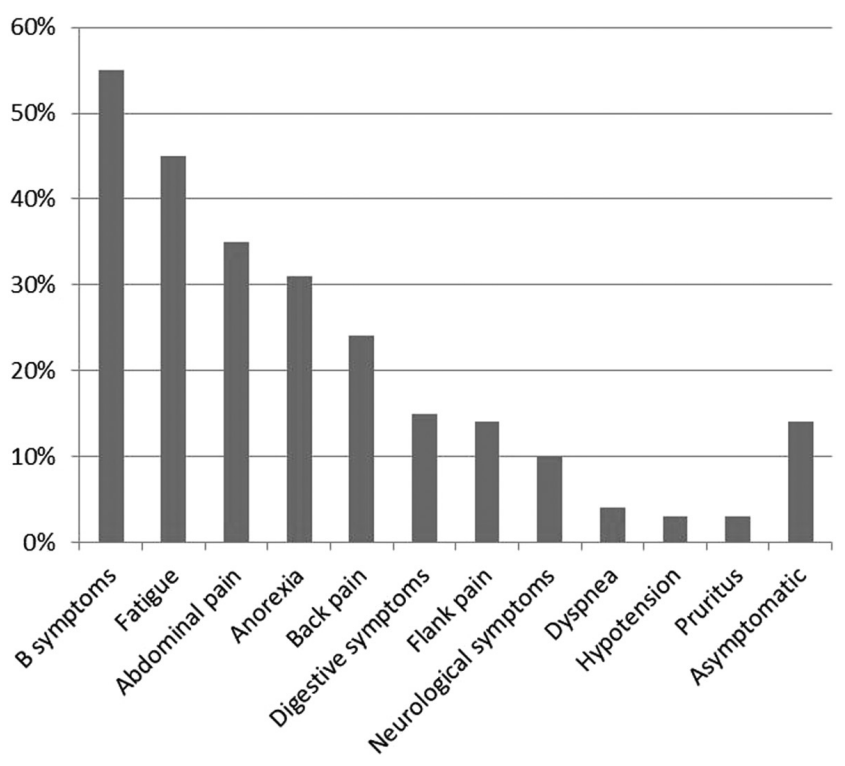

Figure 1

Symptoms of primary adrenal lymphoma (PAL) at diagnosis.

six patients were diagnosed as $\mathrm{T}$ cell lymphoma, two had a NK/T cell lymphoma, and one patient had Hodgkin's lymphoma.

On immunohistochemistry, the B-cell marker CD20 was expressed in 36 of 37 evaluated B cell lymphomas. CD10, BCL2, BCL6 and MUM1 were expressed in 18\% (6/34), 93\% (13/14), 72\% (18/25), and 100\% (17/17) evaluated cases with DLBCL, respectively. All cases classified as DLBCL showed strong expression of the proliferation marker Ki-67 (median 85\%, range 50-100\%), confirming the highly proliferative nature of this lymphoma.

\section{Radiological findings}

CT and PET imaging reports were available for 81 and 18 patients, respectively. Information regarding the size of the adrenal mass was available for 68 patients. The median diameter of the lesion was $80 \mathrm{~mm}$ (range 27-180 $\mathrm{mm})$. Extra-adrenal involvement was detected through radiological examination in $77 \%$ of the patients $(62 / 81)$. The most common extra-adrenal organ manifestations were brain and spleen, followed by involvement of lung, kidney and inferior vena cava. Thirty patients (37\%) had associated lymphadenopathy, nine of them without further extra-adrenal organ involvement. Of the 81 patients, 19 (23\%) had isolated adrenal lymphoma (iPAL). In all patients with available 18-fluorodeoxyglucose (18FDG)-PET ( $n=18)$, standard uptake values (SUV) were elevated in the adrenal glands, with a median SUVmax of
Table 1 Patient characteristics.

\begin{tabular}{l} 
Characteristics \\
\hline Total, $n$ \\
Women \\
Men \\
Age \\
Median \\
Range \\
Lymphoma localization \\
Isolated adrenal involvement \\
Extra-adrenal involvement \\
Data not available \\
Laterality \\
Bilateral \\
Unilateral \\
Data not available \\
LDH \\
Elevation above normal limit \\
Median \\
Range \\
Adrenal insufficiency \\
With bilateral involvement* \\
With unilateral involvement* \\
Unknown/not evaluated \\
Histopathology \\
B cell non-Hodgkin lymphomas \\
Diffuse large B cell lymphoma \\
Mantle cell lymphoma \\
Marginal zone lymphoma \\
Follicular lymphoma \\
B cell lymphoma not otherwise specified \\
Non-B cell lymphoma \\
T cell lymphoma \\
NK/T cell lymphoma \\
Hodgkin lymphoma \\
\hline
\end{tabular}

\begin{tabular}{c}
\hline Values \\
\hline 97 \\
$29(30 \%)$ \\
$68(70 \%)$ \\
66 \\
$25-79$ \\
$19 / 81(23 \%)$ \\
$62 / 81(77 \%)$ \\
16 \\
$44 / 90(49 \%)$ \\
$46 / 90(51 \%)$ \\
7 \\
$53 / 65(81 \%)$ \\
$850 \mathrm{U} / \mathrm{L}$ \\
$174-6515 \mathrm{U} / \mathrm{L}$ \\
$14 / 37(38 \%)$ \\
$14 / 20(70 \%)$ \\
$0 / 17(0 \%)$ \\
60 \\
$88 / 97(91 \%)$ \\
$74 / 97$ \\
$3 / 97$ \\
$2 / 97$ \\
$2 / 97$ \\
$7 / 97$ \\
$9 / 97(9 \%)$ \\
$6 / 97$ \\
$2 / 97$ \\
$1 / 97$ \\
\end{tabular}

* In evaluated patients.

17 (range, 3.5-48). Among patients who had both CT and PET scans, 18FDG-PET revealed extra-adrenal involvement not detected by CT scan in 9/18 cases (50\%). PET-CT images are displayed for four patients who showed a marked response to systemic treatment (Figs 2, 3, 4 and 5).

\section{Treatment}

Data regarding lymphoma treatment was available for 69 patients. Of five patients who died without receiving chemotherapy, two were treated with corticosteroids (dexamethasone and prednisolone, respectively). The other 64 patients received chemotherapy, in four cases (6\%) combined with radiotherapy and in six cases (9\%) as adjuvant therapy after adrenalectomy. The most common first-line chemotherapy protocol was $\mathrm{CHOP}$ (cyclophosphamide, hydroxydaunorubicin, vincristine, and prednisone), given to 53 patients (77\%). One patient with B-cell lymphoma received an intensive 

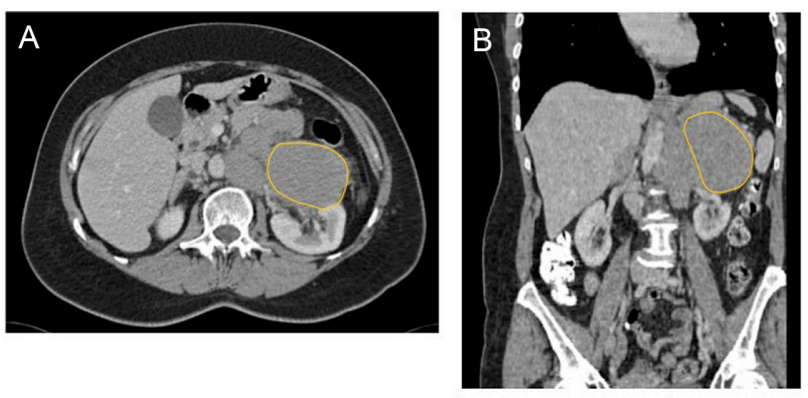

Hyper-CVAD protocol (course A: cyclophosphamide, vincristine, adriamycin and dexamethasone; course B: methotrexate and cytarabine). Another patient, who showed histopathological findings of Hodgkin lymphoma, was treated according to the ABVD protocol (adriamycin, bleomycin, vinblastine, and dacarbazine). In 46 of 61 patients with B-cell lymphomas (75\%), rituximab (R) was part of the treatment regimen. One patient was treated with rituximab and bendamustine during first-line therapy and with R-CHOP after disease progression. Five patients received CNS prophylaxis, of whom two patients received intrathecal methotrexate (MTX); one patient underwent whole brain irradiation; one patient was given high doses of i.v. MTX (two cycles); and another patient

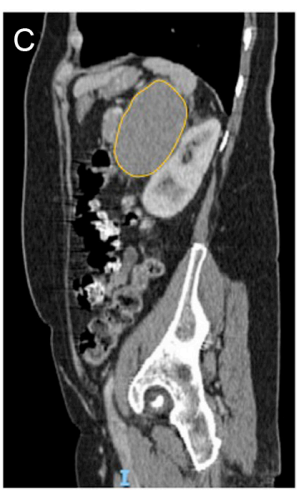

\section{Figure 2}

CT images illustrating unilateral iPAL. CT scans (A, axial plane; B, coronal plane; $C$, sagittal plane) showing a unilateral large $(10.7 \mathrm{~cm})$ left adrenal mass in a 53-year-old female patient who presented with abdominal pain and pruritus. On biopsy, a primary adrenal B-cell lymphoma was diagnosed. received intrathecal triple therapy (MTX, dexamethasone, cytarabine).

Since endocrinological testing of adrenal status, in particular by using the short synacthen test, was only performed in a small fraction of patients $(n=13)$ at the time of diagnosis and follow-up SSTs were not available at all, it was not possible to assess the impact of lymphoma treatment on adrenal status.

\section{Outcomes and prognostic factors}

For 62 patients, response to first-line therapy was evaluable: $34 \%(21 / 62)$ achieved partial remission (PR) and 44\% (27/62) achieved complete remission
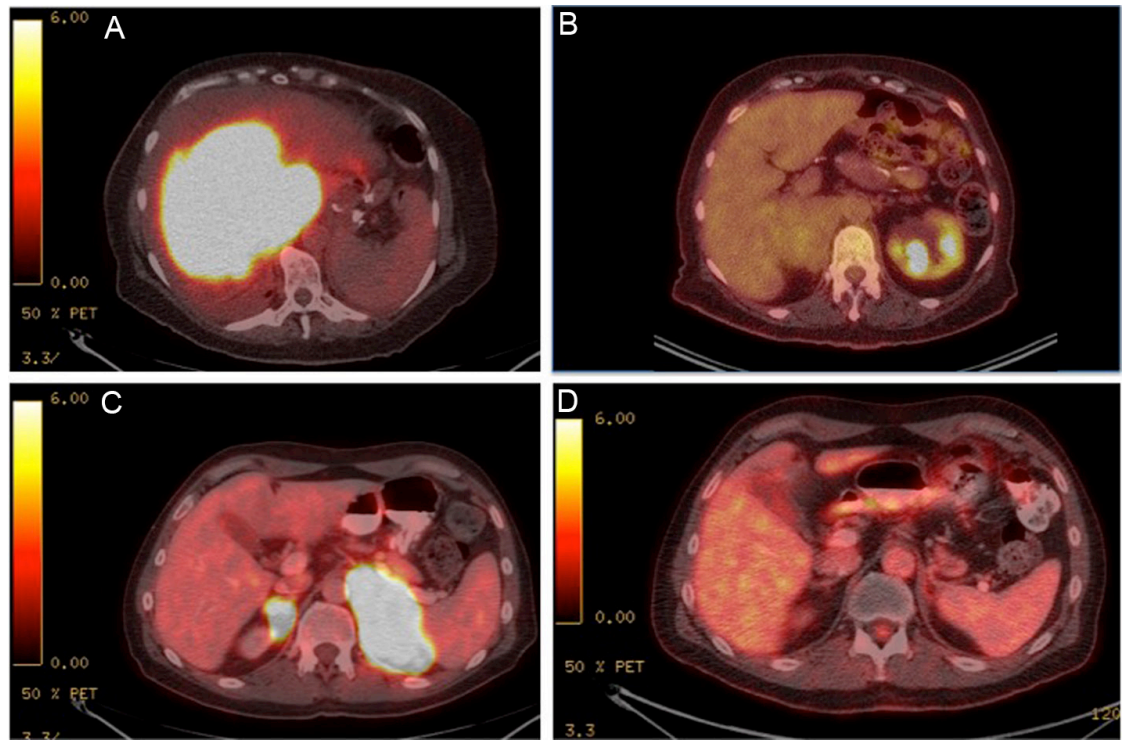

\section{Figure 3}

PET-CT images illustrating unilateral IPAL and bilateral $\mathrm{PAL}$, respectively, at diagnosis and during follow-up after treatment. (A) FDG PET-CT scan showing a large right adrenal mass at diagnosis in a 65-year-old female patient who presented with backpain and B-symptoms. On biopsy, a primary adrenal B-cell lymphoma was diagnosed. (B) The patient responded to six cycles of R-CHOP immunochemotherapy. (C) FDG PET-CT scan showing a bilateral adrenal mass at diagnosis in a 62-year-old male patient with primary adrenal B-cell lymphoma who presented with fatigue, weekness, abdominal pain and confirmed adrenal insufficiency at diagnosis. (D) After treatment failure with R-CHOP, the patient achieved a remarkable remission with the Bruton's tyrosine kinase inhibitor Ibrutinib. 

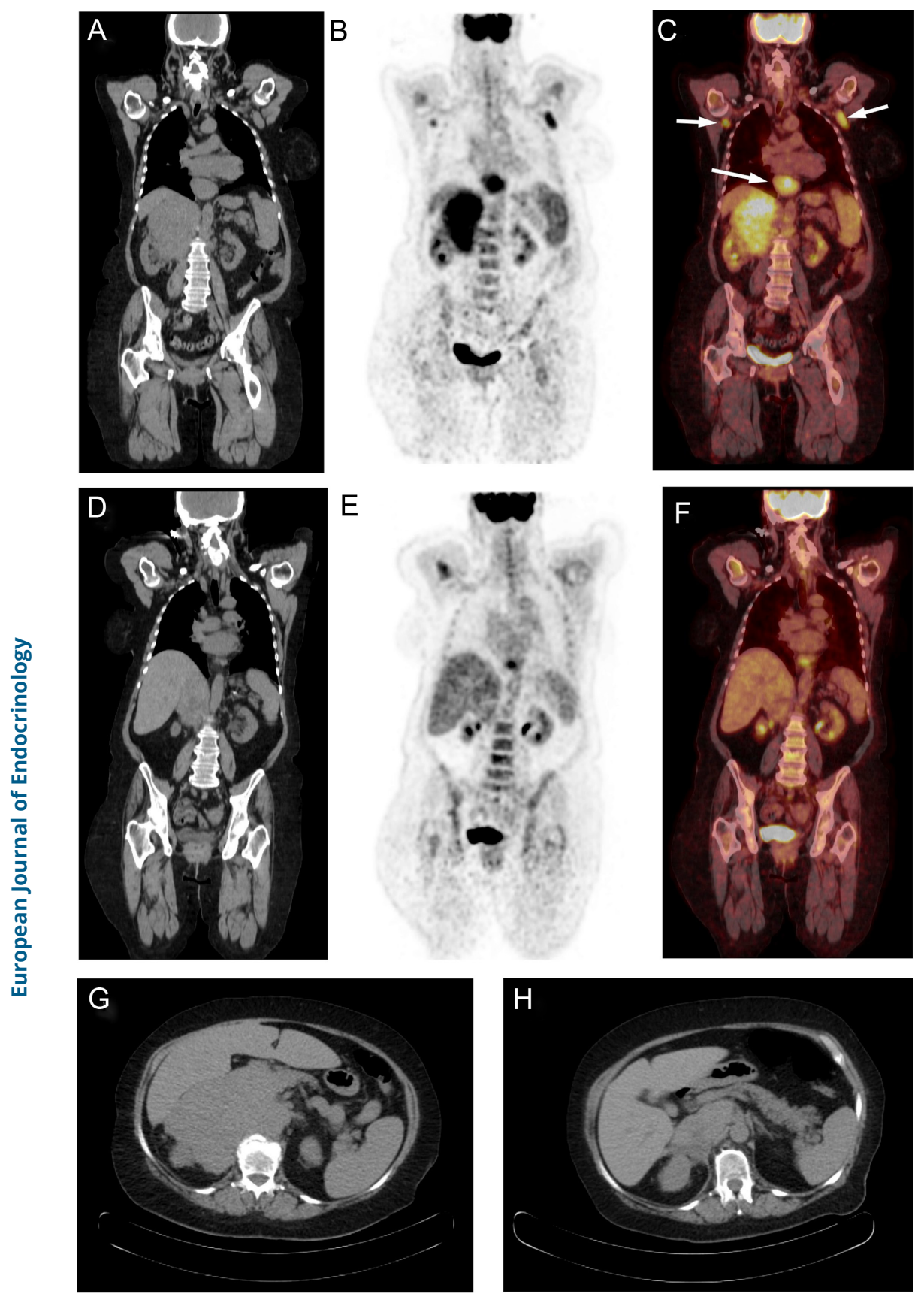

\section{Figure 4}

CT (coronal reconstruction), PET and fusion PET/CT images illustrating unilaterial PAL+ before and after treatment with immuno-chemotherapy. (A, B and C) Prior to treatment, CT, PET and fusion PET/CT showed a unilateral FDG positive large left adrenal mass (14.1 cm, SUV max 9.2) and, in addition, axillary and mesenteric lymphadenopathy (see arrows, SUV max 7.4-8.6) in a 66-year-old female patient who presented with weakness, anorexia and abdominal pain. On biopsy, a primary adrenal B-cell lymphoma was diagnosed. (D, E and F) The patient showed a good partial response to four cycles of R-CHOP immuno-chemotherapy. ( $G$ and H) CT images (axial sections) before and after treatment

(CR). Fourteen of 62 patients (23\%) showed disease progression (DP) during or shortly after first-line therapy. Eighteen of 62 patients (29\%) relapsed after an initial response to treatment. Six patients (three in CR and three with DP after first-line therapy) had secondary involvement of the brain, one of them despite prior CNS prophylaxis using whole brain irradiation.

Median follow-up of patients was 42 months. The 3 -year PFS and OS were 36\% and 39\%, respectively. Median PFS and OS were 10.5 (range 0-120) and 16 (range 0-120) months, respectively (Fig. 6).
We evaluated several potential prognostic factors by univariable analysis (Table 2). B cell lymphomas were associated with better PFS and OS than other lymphoma types. There was a statistically significant association between iPAL and shorter progression-free survival (HR for progression 2.721, 95\% CI 1.280-5.787, P=0.009) (Fig. 7A). Patients with iPAL also appeared to have considerably shorter overall survival (Fig. 7B), but the difference did not reach statistical significance on univariable analysis (HR 1.93, $P=0.11$ ). 

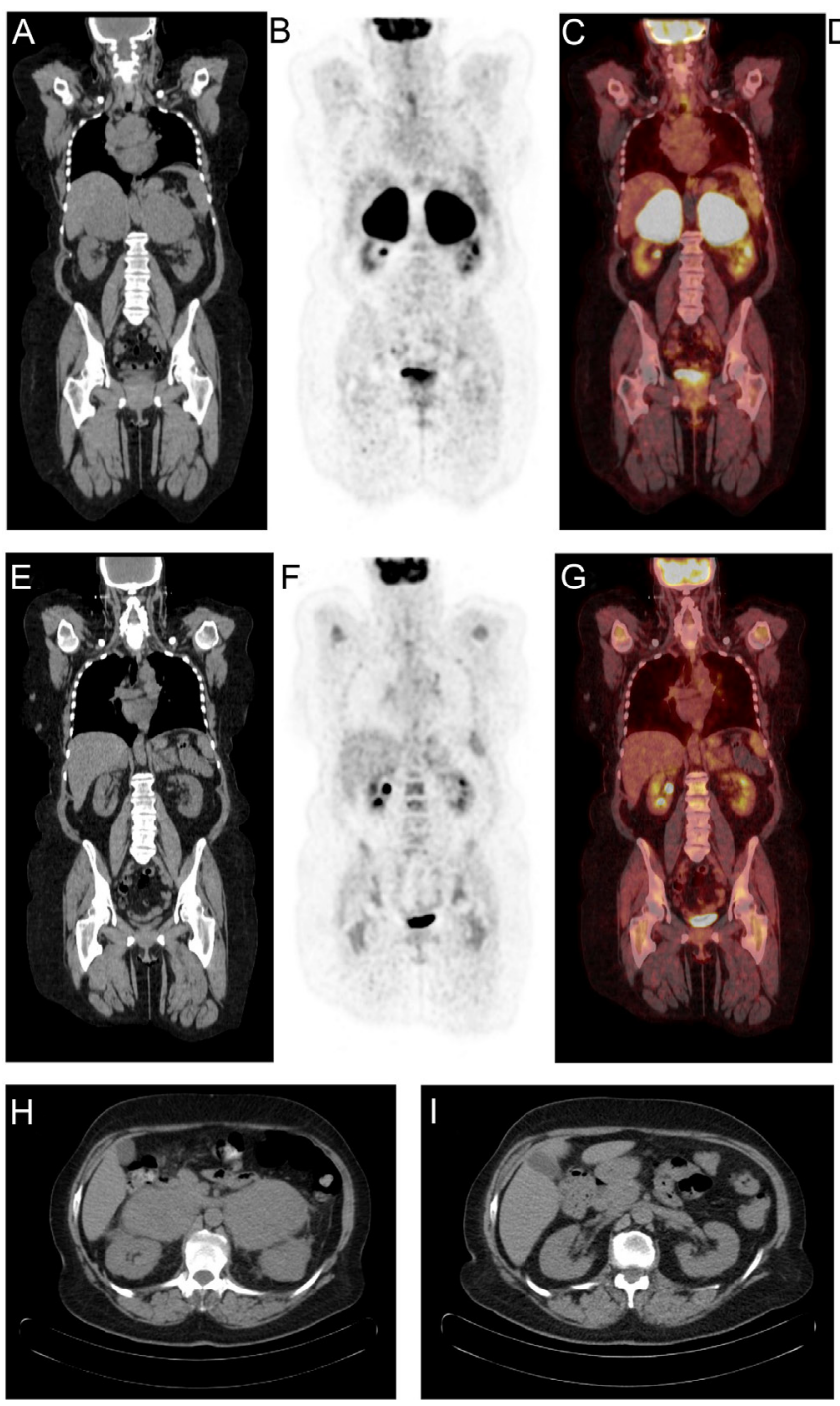

Too many possible confounders precluded formal assessment of treatment effects. However, it was our impression that treatment regimens other than R-CHOP did not substanially alter the outcome. Neither did we observe any substantial differences in treatment regimens between iPAL and PAL+ that may explain the worse outcome in iPAL. Seven responding patients (five in CR and two in PR) received autologous stem cell transplantation. Follow-up information was available for four of these patients, who all showed relatively long overall survival (median 93, range 72-120 months). However, the small number of cases precluded meaningful statistical analysis.

We suspect that the failure to perform adequate endocrinological testing (and subsequent failure to institute cortisol replacement therapy) may have contributed to the bad prognosis in some of the patients. For instance, one of the patients who underwent

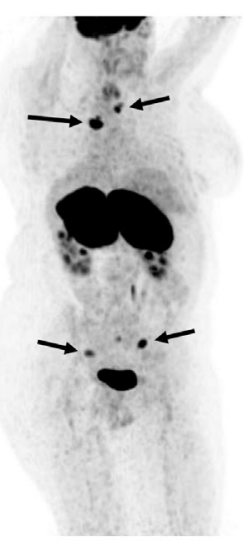

\section{Figure 5}

$\mathrm{CT}$ (coronal reconstruction), PET and fusion PET/CT images illustrating bilaterial $\mathrm{PAL}+$ before and after treatment with immuno-chemotherapy. (A, B, C and D) Prior to treatment, $C T$, PET and fusion PET/CT showed bilateral adrenal masses (SUV max 23.5) plus further extranodal lesions including thyroid, lung, ovary and bones (see arrows in D (PET-MIP), SUV max (right ovary) 5.7-14.8 (Th3)) in a 59-year-old female patient who presented with B-symptoms, anorexia, and abdominal and back pain. (E, $F$ and $G$ ) The patient achieved complete remission after two cycles of cytarabine (pre-phase chemotherapy), four cycles of chemoimmunotherapy with obinutuzumab/ifosfamide/etoposide, followed by two cycles of high-dose methotrexate (for CNS prophylaxis). ( $\mathrm{H}$ and I) CT images (axial sections) before and after treatment.

autologous stem cell transplantation without prior endocrinological testing died soon after the procedure, with no clear cause of death ascertained. This patient may have suffered from adrenal insufficiency, which may have made it impossible for him to tolerate the stresses and strains of the procedure.

\section{Discussion}

Primary adrenal lymphoma (PAL) was first described in 1961 (8). With fewer than 250 published cases in the medical literature, there are limited data regarding the epidemiology, clinical features, and pathophysiology of this rare disease. Our current retrospective analysis of 97 patients from 14 centers is among the largest surveys so far. Expectedly, the heterogeneity of the reported investigations and treatments, as well as the problem of 

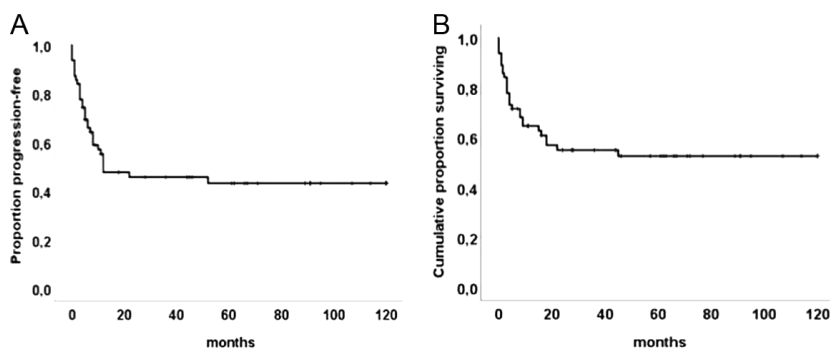

\section{Figure 6}

Kaplan-Meier estimates of progression-free survival (A) and overall survival $(B)$ in patients with primary adrenal lymphoma.

missing data, places limitations on our retrospective study. Therefore, we tried to avoid unsubstantiated conclusions, for instance, regarding the efficacy of various treatment regimens. The lack of adequate endocrinological assessment in a large proportion of patients must also be considered a limiting factor. This inadequacy, however, reflects the real-life situation of patient care for PAL, which is in need of improvement.

Regarding our distinction between PAL and PAL+, the respective diagnoses obviously depend on diagnostic imaging. It would have been desirable to have FDG-PET scans available in the majority of patients. However, due to the retrospective nature of our study, this was not possible. Nevertheless, absence of 18F-FDG PET in the majority of our retrospective cases is a limitation of the study, since we cannot exclude with certainty that PET might have detected a few additional extra-adrenal manifestations.

Based on 18 evaluable patients, our study suggests that PET is superior to CT scan in the detection of extraadrenal manifestations. We found that, among patients who had both CT and PET scans, FDG-PET revealed extraadrenal involvement not detected by CT scan in 9/18 cases (50\%). All of these extra-adrenal manifestations presented as enlarged lymph nodes. Although the quality of tomographic imaging studies has improved over the years, older CT scans were of sufficient quality to detect large adrenal tumors as well as substantial lymphadenopathy. Our conclusion that PET-CT appears to be superior to CT regarding the detection of extra-adrenal involvement is based on patients who had both CT and PET scans within a relatively short period of time. This intra-individual comparison avoids comparing recent PET-CT technology with older versions of CT scanning.

Novel, clinically relevant prognostic factors are needed for PAL because current lymphoma staging systems and prognostic scores may not be reliable. This is illustrated by our finding that, in contrast to recent publication on primary adrenal lymphoma by Li et al. (9), increased tumor burden in the form of bilateral adrenal involvement or large tumor diameter had no impact on prognosis.

We presume that, in the future, knowledge of the mutational landscape of PAL may aid in the prognostic assessment of patients. Recently, Chapuy et al. showed that primary CNS lymphoma (PCNSL) and primary testicular lymphoma (PTL) have a mutational landscape and immunohistochemical appearance that differ from nodal diffuse large B-cell lymphoma. For instance, the extranodal lymphomas harboured more numerous somatic mutations and showed more frequent overexpression of the PD-1 ligand (10). Nothing is known about the corresponding features of PAL. Therefore, it is also difficult to predict whether modern lymphoma therapies, like those targeting the B cell receptor pathway, will be more successful than conventional immunochemotherapy.

In order to improve the treatment of $\mathrm{PAL}$, prompt endocrinological management is as important as effective cancer therapy. In a systematic review by Rashidi et al., $70 / 115(61 \%)$ of evaluated patients had either relative or absolute adrenal insufficiency (2). Of concern, endocrinological assessment was performed in less than $40 \%$ of our cases and just $44.4 \%$ of patients with bilateral

Table 2 Factors influencing PFS and OS according to univariable analysis.

\begin{tabular}{|c|c|c|c|c|c|c|c|}
\hline \multirow[b]{2}{*}{ Variables } & \multirow[b]{2}{*}{ n/total } & \multicolumn{3}{|c|}{ PFS } & \multicolumn{3}{|c|}{ os } \\
\hline & & $\mathrm{HR}$ & $95 \% \mathrm{Cl}$ & $P$ & $\mathrm{HR}$ & $95 \% \mathrm{Cl}$ & $P$ \\
\hline Age $<65$ & $33 / 97$ & 0.370 & $0.159-0.864$ & 0.022 & 0.476 & $0.198-1.143$ & 0.097 \\
\hline Diameter $>8 \mathrm{~cm}$ & $36 / 68$ & 1.136 & $0.423-1.832$ & 0.733 & 0.994 & $0.469-2.106$ & 0.987 \\
\hline Bilateral adrenal involvement & $44 / 90$ & 0.755 & $0.664-2.643$ & 0.426 & 0.684 & $0.320-1.462$ & 0.327 \\
\hline $\mathrm{iPAL}$ & $19 / 81$ & 2.721 & $1.280-5.787$ & 0.009 & 1.920 & $0.863-4.273$ & 0.110 \\
\hline $\mathrm{LDH}>250 \mathrm{U} / \mathrm{L}$ & $53 / 65$ & 1.244 & $0.465-3.327$ & 0.664 & 1.272 & $0.477-3.390$ & 0.631 \\
\hline Adrenal insufficiency & $14 / 37$ & 0.809 & $0.243-2.690$ & 0.730 & 0.927 & $0.352-2.439$ & 0.878 \\
\hline B cell lymphoma & $88 / 97$ & 0.276 & $0.118-0.646$ & 0.003 & 0.335 & $0.135-0.832$ & 0.018 \\
\hline
\end{tabular}

https://eje.bioscientifica.com 

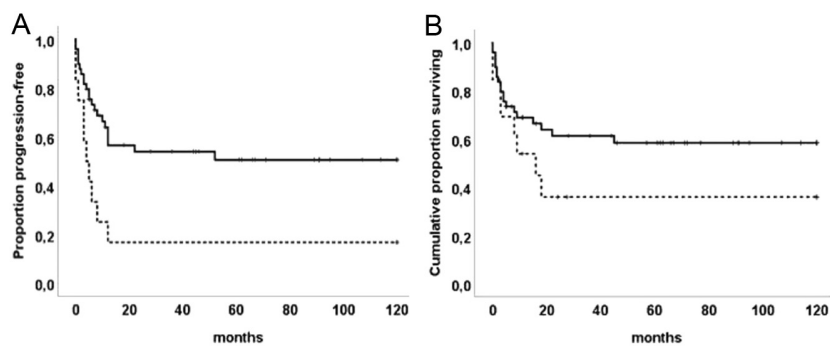

\section{Figure 7}

Kaplan-Meier estimates of progression-free survival (A) and overall survival (B) in patients with iPAL (isolated adrenal lymphoma involvement; dotted lines) and PAL+ (featuring additional extraadrenal manifestation; solid lines).

involvement. In most of the patients, adrenal insufficiency was only assessed by baseline cortisol measurement, which helps ruling out adrenal insufficiency only if a level is above a value that different studies placed between $285 \mathrm{nmol} / \mathrm{L}(10.3 \mu \mathrm{g} / \mathrm{dL})$ and $>480 \mathrm{nmol} / \mathrm{L}(17 \mu \mathrm{g} / \mathrm{dL})$ (11). Although destruction of $90 \%$ of the adrenal glands is required for the development of clinical signs and symptoms of adrenal insufficiency, subclinical adrenal insufficiency may be present earlier, due to cytokinedriven, paracrine effects of lymphoma cells on the adrenal gland microenvironment (2). This degree of adrenal insufficiency would not be detected by simply measuring baseline cortisol, even if performed in the morning, since the result may fall within the normal range. Inadequate adrenal reserve may become a clinical problem in case of severe illness, surgical intervention or in the context of corticosteroid withdrawal after immunochemotherapy (12). It is thus necessary not only to be vigilant regarding signs and symptoms of adrenal insufficiency, including skin hyperpigmentation, fatigue, hypotension, and electrolyte abnormalities, but also to conduct a formal assessment of adrenal reserve. Failure to do so may have contributed to the worse prognosis in our patients with iPAL. It is possible that adrenal insufficiency was at least partially responsible for the early demise of six of nine patients with iPAL whose death was not attributable to progressive lymphoma but to adrenal crisis, sepsis, poor general condition, or adrenalectomy.

In conclusion, our retrospective analysis suggests that PAL is a heterogeneous disease and comprises cases with isolated involvement of adrenal tissue (iPAL) and cases with additional extra-adrenal organ manifestations $(\mathrm{PAL}+)$. It is possible that iPAL, which has an unusual male/female ratio, less $\mathrm{B}$ symptoms, and a particularly poor prognosis, may have biological characteristics that are associated with a pronounced tendency for early destruction of adrenal endocrine tissue and subsequent adrenal insufficiency. Accordingly, we emphasize the need for careful endocrine assessment of patients with PAL and for close cooperation between oncologists and endocrinologists when treating patients with this rare disease.

\section{Declaration of interest}

$\mathrm{M} \mathrm{F}$ is an associate editor and W A is Editor in Chief for the European Journal of Endocrinology. However, they were not involved in any way in the review or editorial process for this paper, on which they are listed as authors. The other authors have nothing to disclose.

\section{Funding}

W A receives support from the National Institute for Health Research (NIHR) Birmingham Biomedical Research Centre at the University Hospitals Birmingham NHS Foundation Trust and the University of Birmingham (Grant Reference Number BRC-1215-20009). The views expressed are those of the author(s) and not necessarily those of the NIHR or the Department of Health and Social Care. V C receives support from the Academy of Medical Sciences UK (Starter Grant for Clinical Lecturers SGL020/1018).

\section{References}

1 Laurent C, Casasnovas O, Martin L, Chauchet A, Ghesquieres H, Aussedat G, Fornecker LM, Bologna S, Borot S, Laurent K et al. Adrenal lymphoma: presentation, management and prognosis: presentation. QJM 2017110 103-109. (https://doi.org/10.1093/ qjmed/hcw174)

2 Rashidi A \& Fisher SI. Primary adrenal lymphoma: a systematic review. Annals of Hematology 201392 1583-1593. (https://doi. org/10.1007/s00277-013-1812-3)

3 Kim YR, Kim JS, Min YH, Hyunyoon D, Shin HJ, Mun YC, Park Y, Do YR, Jeong SH, Park JS et al. Prognostic factors in primary diffuse large B-cell lymphoma of adrenal gland treated with rituximabCHOP chemotherapy from the Consortium for Improving Survival of Lymphoma (CISL). Journal of Hematology and Oncology 2012549. (https://doi.org/10.1186/1756-8722-5-49)

4 Spyroglou A, Schneider HJ, Mussack T, Reincke M, von Werder K \& Beuschlein F. Primary adrenal lymphoma: 3 case reports with different outcomes. Experimental and Clinical Endocrinology and Diabetes 2011119 208-213. (https://doi. org/10.1055/s-0031-1271629)

5 Bancos I, Tamhane S, Shah M, Delivanis DA, Alahdab F, Arlt W, Fassnacht M \& Murad MH. DIAGNOSIS OF ENDOCRINE DISEASE: The diagnostic performance of adrenal biopsy: a systematic review and meta-analysis. European Journal of Endocrinology $2016 \mathbf{1 7 5}$ R65-R80. (https://doi.org/10.1530/EJE-16-0297)

6 Babinska A, Peksa R \& Sworczak K. Primary malignant lymphoma combined with clinically 'silent' pheochromocytoma in the same adrenal gland. World Journal of Surgical Oncology 201513289. (https://doi.org/10.1186/s12957-015-0711-6)

7 Fassnacht M, Arlt W, Bancos I, Dralle H, Newell-Price J, Sahdev A, Tabarin A, Terzolo M, Tsagarakis S \& Dekkers OM. Management of adrenal incidentalomas: European Society of Endocrinology Clinical Practice Guideline in collaboration with the European Network for the Study of Adrenal Tumors. European Journal of Endocrinology 2016 175 G1-G34. (https://doi.org/10.1530/EJE-16-0467) 
8 Hayes JA \& Christensen OE. Primary adrenal lymphoma. Journal of Pathology and Bacteriology 196182 193-194. (https://doi.org/10.1002/ path.1700820124)

9 Li S, Wang Z, Wu Z, Zhuang H \& Xu Y. Clinical characteristics and outcomes of primary adrenal diffuse large B cell lymphoma in a large contemporary cohort: a SEER-based analysis. Annals of Hematology 201998 2111-2119. (https://doi.org/10.1007/s00277019-03740-9)

10 Chapuy B, Roemer MG, Stewart C, Tan Y, Abo RP, Zhang L, Dunford AJ, Meredith DM, Thorner AR, Jordanova ES et al. Targetable genetic features of primary testicular and primary central nervous system lymphomas. Blood 2016127 869-881. (https://doi. org/10.1182/blood-2015-10-673236)

11 Bornstein SR, Allolio B, Arlt W, Barthel A, Don-Wauchope A, Hammer GD, Husebye ES, Merke DP, Murad MH, Stratakis CA et al. Diagnosis and treatment of primary adrenal insufficiency: an Endocrine Society clinical practice guideline. Journal of Clinical Endocrinology and Metabolism 2016101 364-389. (https://doi. org/10.1210/jc.2015-1710)

12 Carvalho F, Louro F \& Zakout R. Adrenal insufficiency in metastatic lung cancer. World Journal of Oncology 20156 375-377. (https://doi. org/10.14740/wjon890w)

Received 2 July 2019

Revised version received 31 May 2020

Accepted 16 June 2020 